\title{
A Blind Source Separation Method Using Denoising Strategy Based on ICEEMDAN and Improved Wavelet Threshold
}

\author{
Lu Feng ${ }^{D}$, Jiong Li $(\mathbb{D}$, Changqing Li, and Yang Liu \\ School of Space Information, Space Engineering University, Beijing 101416, China \\ Correspondence should be addressed to Jiong Li; lij_2015@126.com
}

Received 26 November 2021; Revised 29 January 2022; Accepted 31 January 2022; Published 28 February 2022

Academic Editor: Paolo Crippa

Copyright (C 2022 Lu Feng et al. This is an open access article distributed under the Creative Commons Attribution License, which permits unrestricted use, distribution, and reproduction in any medium, provided the original work is properly cited.

\begin{abstract}
Traditional blind source separation (BSS) methods often want no additional noise effects. But in practice, noise is ubiquitous, and there are even cases with low signal-to-noise ratios (SNR). Unfortunately, these blind source separation methods have a significant impact on system performance in the presence of noise. This study proposes a blind source separation method to eliminate noise and extract signal information to the maximum extent. The method is based on improved complete ensemble empirical mode decomposition with adaptive noise (ICEEMDAN) and improved wavelet threshold (IWT) hierarchical decomposition to improve the signal separation effect. First, the signal is decomposed via ICEEMDAN to obtain the noise-dominated and informationdominated intrinsic mode function (IMF) components. Then, the noise-dominated IMF components are again decomposed by ICEEMDAN, and each component's scale function is obtained via detrended fluctuation analysis (DFA). Next, the noisedominated IMF components generated in the second decomposition round undergo an improved wavelet threshold denoising to obtain useful signal information. Finally, the information-dominated IMF components generated in the two rounds of decomposition and the resulting signal of wavelet denoising yield a reconstructed signal, which is then decomposed using the BSS algorithm to obtain the source signal. The experimental results demonstrate that the proposed method can accurately extract signal information and can better separate the source signal than the existing algorithm.
\end{abstract}

\section{Introduction}

Blind source separation aims to recover a source signal from a mix of signals in a transmission channel. The technique employs separation criteria based on the analysis of the observed signal's time-frequency, statistical, and other characteristics and is suitable for scenarios where the source signals and transmission channel parameters are unknown $[1,2]$. BSS algorithms are widely used in domains such as digital image processing [3], speech signal recognition [4, 5], magnetic resonance imaging of the brain [6], and remote sensing image processing [7]. Nevertheless, these applications typically ignore the noise impact on the system or regard the noise impact as small. However, certain communication systems (e.g., satellite communication systems) are sensitive to noise, thus preventing a direct application of BSS algorithms. The satellite communication system is a typical wireless communication system with a low signal-to- noise ratio, and a large variety of noises impact the accuracy of its signal reception. Research shows that the signal separation performance decreases significantly when the SNR is lower than $10 \mathrm{~dB}$ [8]. Therefore, there is a need to explore methods that can achieve high BSS performance in the presence of noise.

In recent years, scholars have put forward numerous theories and methods for BSS under noisy conditions, and the more common method is to combine the noise reduction algorithm with the BSS algorithm. At present, the mainstream noise reduction algorithms include multivariable time series [9], K-means [10], stochastic resonance [11], multiobjective evolutionary algorithms [12], wavelet transform, and Hilbert transform [13]. For example, Lei et al. [14] proposed a method that combines wavelet threshold denoising and independent component analysis (ICA) to separate noisy mixed speech signals. Independent component analysis and canonical correlation analysis (CCA) are 
widely used as two methods for noise removal of HD-sEMG signals, and Wang et al. [15] proposed a new method for noise removal based on independent vector analysis, which significantly improved the system performance. Snoussi and Idier [16] employed the generalized hyperbolic $(\mathrm{GH})$ process to model the signal source and proposed a Bayesian sampling solution. The algorithm achieves good results in the cases of low SNR and underdetermined mixing. Regarding single-channel denoising, Hongyan and Guanglong [17] proposed a noisy speech signal separation method based on the single-channel ICA speech enhancement algorithm and the FastICA algorithm. Gu et al. [18] modeled the distribution of signal sources via the Gaussian mixture model (GMM) by using the non-Gaussian nature of signal sources. The expectation-maximization algorithm obtains the maximum a posteriori probability estimates of the mixture matrix, GMM parameters, and noise covariance matrix. Building on the receiver's a priori knowledge, Weiss and Yeredor [19] proposed an iterative method for solving the source signal's minimum mean square error based on maximum likelihood estimation. The discussed methods perform model fitting to recover source signals, which requires a considerable amount of a priori knowledge on source signals and runs a risk of achieving poor performance due to insufficient adaptability of the algorithm.

Empirical mode decomposition (EMD) is a signal processing method proposed by Huang et al. [20] for processing nonlinear and nonstationary signals. EMD decomposes a signal into multiple intrinsic mode functions with different frequencies and bandwidths according to the signal's time scale characteristics. It is an adaptive decomposition method that requires no a priori information. Due to this advantage, many EMD denoising algorithms have been proposed. Using the fact that higher-order statistics of Gaussian white noise are zero, Tsolis and Xenos [21] proposed a method to detect whether there is only Gaussian white noise in an IMF component. In real-world applications, however, noisy signal sampling points are very limited, and calculating the higherorder statistics using such limited sampling points may introduce large errors. Inspired by translation invariant wavelet threshold, Kopsinis and McLaughlin [22] proposed an interval threshold algorithm based on EMD, ensuring the reconstructed signal's continuity. Qian et al. [23] combined DFA with EMD, where DFA generates a threshold function for separating IMF components.

The listed EMD-based denoising methods mostly obtain the dividing point between noise-dominated IMF components and information-dominated IMF components by calculating the relationship between the threshold function and the threshold. Furthermore, the methods discard the noisedominated IMF components before reconstructing the signal. In real-world applications, however, certain signal information is contained in noise-dominated IMF components. Therefore, directly discarding these components affect the system's performance. To solve this problem, Zhou et al. [24] proposed a hierarchical denoising strategy (EMD-HMA) based on EMD. According to this strategy, after the noisedominated IMF components are distinguished from the information-dominated IMF components, the noise-dominated
IMF components are decomposed once again to extract the signal information further. However, the algorithm achieves only a limited improvement in simulation analysis. To further promote the denoising performance and the signal information retainment, this study proposes a BSS method featuring a hierarchical decomposition and denoising strategy based on ICEEMDAN and IWT denoising.

The main contributions of this study are summarized as follows:

(i) As an adaptive data-driven approach, ICEEMDAN has more precise and reliable performance than EMD and its extension methods while overcoming their problems

(ii) As a dividing point calibration method for noisedominated IMF components and informationdominated IMF components, the DFA method better solves the calibration fuzzy problem

(iii) We propose a strategy for suppressing noise-based hierarchical decomposition, which can maximize the extraction of signal information through secondary decomposition and further IWT denoising.

The rest of this study is organized as follows. Section 2 is the introduction of prior knowledge, including the BSS algorithm, ICEEMDAN algorithm, DFA algorithm, and improved wavelet threshold function. The BSS method featuring a hierarchical decomposition and denoising strategy is proposed in Section 3. The computer numerical simulation results are presented in Section 4. Finally, a conclusion is drawn in Section 5.

\section{Prior Knowledge}

2.1. BSS Algorithm. Independent component analysis (ICA) is a classical BSS method based on the statistical independence of source signals. Since communication signals originate from different transmitting sources, they are statistically independent and, therefore, satisfy the ICA conditions.

The system model under noisy conditions is

$$
X=A S+N,
$$

where $S=\left[S_{1}(t) S_{2}(t)\right]^{T}$ denotes the source signal, $X=\left[X_{1}(t) X_{2}(t)\right]^{T}$ is the observed signal, $A$ is the full rank mixing matrix, and $N$ denotes the additive Gaussian white noise. BSS aims to find a separation matrix $W$ and process the received signal as

$$
Y=W X
$$

such that $Y$ is an effective estimate of the source signal $S$. However, BSS cannot achieve complete identification of the separation matrix $W$. There are two uncertainties in BSS:

(1) Scale uncertainty: since the transmission system parameters and the source signals are unknown, the observed signal $X$ may be the interaction result of the mixing matrix $A$ and the source signal $S$ at different scales. However, the BSS algorithm does not limit the 
separation matrix scale, resulting in a scale difference between the estimated signal $Y$ and the source signal $S$.

(2) Order uncertainty: switching the positions of the source signal $S$ and the corresponding position in the mixing matrix $A$ yields an identical observation signal $X$. Therefore, the order of the signals cannot be determined after separation.

2.2. ICEEMDAN Algorithm. Since its proposal, the EMD algorithm has been widely used in nonlinear and nonstationary signal processing problems. However, the EMD's mode mixing problem greatly affects the algorithm's performance. To overcome the problems regarding long calculation time, residual noise, and mode mixing, $\mathrm{Wu}$ et al. proposed ensemble empirical mode decomposition (EEMD) [25]. However, the method introduces noise during signal decomposition, thus producing a distortion in signal reconstruction. Yeh et al. aimed to eliminate this reconstruction error in EEMD and, in 2010, proposed a complementary EEMD (i.e., CEEMD) [26], which adds noise to the original data in pairs. However, although CEEMD significantly reduces the residual noise in the reconstructed signal, there is no guarantee that the same number of modes can be generated. In 2011, Torres et al. proposed the complete EEMD with the adaptive noise (i.e., CEEMDAN) [27] algorithm to address this problem. The CEEMADN algorithm has been shown to achieve better results in underwater acoustic signal processing [28, 29]. However, several "pseudo" modes appear in the early stage of this algorithm's decomposition process. The pseudomode problem was effectively solved in 2014 when Colominas et al. proposed the improved CEEMDAN (i.e., ICEEMDAN) [30]. The ICEEMDAN algorithm's steps are as follows:

(1) Calculate the mean value of results produced via EMD in $I$ rounds, obtaining the first residual:

$$
r_{1}=\frac{1}{I} \sum_{i=1}^{I} M\left(x+\varepsilon_{0} \omega^{(i)}\right) .
$$

Here, $\omega^{(i)}$ is the Gaussian white noise whose $i^{\text {th }}$ average value and unit variance are zero, $\varepsilon_{0}$ denotes the set SNR, and $M(\cdot)$ is the operator for calculating the mean value using the EMD algorithm.

(2) In the first stage $(k=1)$, calculate the first mode:

$$
\widetilde{\mathrm{MF}}_{1}=x-r_{1} \text {. }
$$

(3) In the second stage $(k=2)$, estimate the second residual and obtain the second mode:

$$
\begin{aligned}
r_{2} & =\frac{1}{I} \sum_{i=1}^{I} M\left(r_{1}+\varepsilon_{1} E_{2}\left(\omega^{(i)}\right)\right), \\
\widetilde{\mathrm{IMF}_{2}} & =r_{1}-r_{2} \\
& =r_{1}-\frac{1}{I} \sum_{i=1}^{I} M\left(r_{1}+\varepsilon_{1} E_{2}\left(\omega^{(i)}\right)\right) .
\end{aligned}
$$

Here, $E_{j}(\cdot)$ is the operator for generating the $j^{\text {th }}$ mode using the EMD algorithm.

(4) For $k=3, \ldots, K$, calculate the $k^{\text {th }}$ residual:

$$
r_{k}=\frac{1}{I} \sum_{i=1}^{I} M\left(r_{k-1}+\varepsilon_{k-1} E_{k}\left(\omega^{(i)}\right)\right) .
$$

(5) Calculate the $k^{\text {th }}$ mode:

$$
\widetilde{\mathrm{IMF}}_{k}=r_{k-1}-r_{k} \text {. }
$$

(6) Continue filtering until the resulting residual' no further decompositions can be performed (i.e., until less than three local extrema are found):

$$
R=x-\sum_{k=1}^{K} \mathrm{IMF}_{k}
$$

where $R$ is the final residual, and $K$ is the total number of IMF components. Therefore, the signal $x$ is represented as

$$
x=R+\sum_{k=1}^{K} \mathrm{IMF}_{k} .
$$

2.3. DFA Algorithm. The DFA algorithm [31] is a long-term power-law relationship technique. It was first proposed by Peng et al. in 1994 for DNA research, but it is also applicable to the nonstationary signals' processing. The DFA algorithm can better solve the calibration ambiguity problem, so the algorithm can obtain the critical values of noise-dominated and information-dominated IMF components. The specific steps are as follows:

(1) Calculate the mean value of time series $x(i)$ in a given interval $[1, N]$ and perform mean subtraction on the time series $x(i)$ :

$$
\begin{aligned}
\bar{x} & =\frac{1}{N} \sum_{i=1}^{N} x(i), \\
y(k) & =\sum_{i=1}^{k}[x(i)-\bar{x}], \quad k=1,2,3, \ldots .
\end{aligned}
$$

Here, time series $y(k)$ is divided into $[N / n]$ subspace series segments, $[\cdot]$ represents the rounding function, and $n$ denotes the interval length.

(2) The polynomial fitting trend of each subinterval is

$$
y_{s}(i)=\sum_{n=0}^{k} a_{n} i^{n} .
$$

(3) When the fluctuation trend of each subinterval is removed, the volatility variance of each subinterval is expressed as

$$
F^{2}(n, s)=\frac{1}{n} \sum_{i=1}^{n}\left(y[(s-1) n+i]-y_{s}(i)\right)^{2} .
$$


The second-order fluctuation coefficient of the segment series is expressed as

$$
F_{q}(n)=\left[\frac{1}{N_{n}} \sum_{s=1}^{N_{n}} F^{2}(n, s)\right]^{1 / 2} .
$$

(4) Changing the subinterval length $n$ in step (1) and repeating steps (2) and (3) to obtain the time series fluctuation curve, one derives the Hurst index of the time series $x(i)$ :

$$
\begin{aligned}
H & =\frac{\log _{2} F_{q}(n)}{\log _{2} n} \\
& =\frac{\log _{2}\left[1 / N_{n} \sum_{s=1}^{N_{n}} F^{2}(n, s)\right]^{1 / 2}}{\log _{2} n} .
\end{aligned}
$$

(5) The relationship between scale function $\alpha$ and fluctuation function $F(n)$ is

$$
F(n) \propto n^{\alpha} \text {. }
$$

Steps (4) and (5) show that the scaling function $\alpha$ is related to the Hurst index. In this study, the values of the two items are set to be equal. When $0<\alpha<0.5$, the component is the noise-dominated component. Furthermore, $\alpha=0.5$ indicates that the component is irrelevant to the signal, and finally, when $\alpha>0.5$, the component is information-dominated [32]. Thus, by comparing the IMF components' $\alpha$ values, noise-dominated IMF components can be distinguished from information-dominated IMF components.

2.4. Improved Wavelet Threshold Function. In order to solve the problem of signal information loss caused by the ICEEMDAN method, wavelet threshold function is introduced to denoise the noise-dominant IMF components, so as to extract the useful information. The traditional wavelet threshold function mainly includes hard threshold function and soft threshold function. However, the hard threshold function has the problem of discontinuity at the threshold $\lambda$, so there will be local oscillation in signal reconstruction. The soft threshold function is smoothed, but there is a problem of large deviation after signal reconstruction. Applying wavelet threshold function directly to the EMD algorithm significantly affects the reconstructed signal's continuity [24]. Therefore, on the basis of integrating wavelet threshold function and the EMD algorithm, Wang et al. [33] proposed an improved wavelet threshold function, which combines the advantages of hard threshold function and soft threshold function to further reduce the reconstruction error.

The improved wavelet threshold function is expressed as

$$
\widehat{\Psi}_{j, k}= \begin{cases}\operatorname{sign}\left(\Psi_{j, k}\right)\left[\left|\Psi_{j, k}\right|^{2}-\left(\lambda e^{-\left(\left|\Psi_{j, k}\right|-\lambda\right) / k}\right)^{2}\right]^{1 / 2}, & \left|\Psi_{j, k}\right| \geq \lambda, \\ \alpha \Psi_{j, k}, & \left|\Psi_{j, k}\right|<\lambda,\end{cases}
$$

where $\Psi_{j, k}$ is the wavelet coefficients obtained after the wavelet transform, and $\lambda$ is the threshold. Both $k(k>0)$ and the so-called "scaling index" $\alpha(\alpha \in(0.05,0.5))$ can be adjusted according to the actual situation. As $k \longrightarrow 0$, the threshold function is closer to the hard threshold function, whereas when $k \longrightarrow \infty$, the threshold function is closer to the soft threshold function. Similarly, the larger the scaling index $\alpha$, the smoother the signal's potential trend. When $\Psi_{j, k} \longrightarrow \lambda$, the improved wavelet threshold function is continuous at the threshold $\lambda$. When $\Psi_{j, k} \longrightarrow \infty$, the improved wavelet threshold function is an asymptote, and the reconstructed signal is closer to the actual signal.

The threshold $\lambda$ is expressed as

$$
\lambda_{i}=\frac{\delta \sqrt{2 \ln (N)}}{\ln (i+1)},
$$

where $\delta$ represents the noise intensity, $i$ is the number of decomposition layers, and $N$ is the signal length. Threshold $\lambda$ decreases with the increase in the number of decomposition layers.

\section{BSS Method Featuring Hierarchical Decomposition and Denoising Strategy Based on ICEEMDAN and Improved Wavelet Threshold}

In the decomposition process of traditional EMD-based denoising algorithms [34], the first IMF component is usually dominated by noise. Therefore, it has minimum signal information and is usually discarded to suppress noise. Although several methods successfully distinguish noise-dominated IMF components from informationdominated ones, discarding noise-dominated IMF components deletes the signal information contained therein, which will undoubtedly affect the signal separation performance.

Hierarchical multiresolution analysis is to zoom the signal in a particular frequency band by representing the signal in that particular frequency band as the sum of next level components [35]. The method of EMD-based hierarchical multiresolution analysis can further zoom the intrinsic time scales from coarse level to fine level. Since the detail information of the noise-dominated IMF is obtained from a further decomposition level, better denoising performance can be achieved [24]. However, EMD has the problems of long calculation time, residual noise, and mode aliasing. Therefore, we use the ICEEDMDAN algorithm to replace the EMD algorithm and hope to further decompose the noise-dominated IMF components generated in the first decomposition round and perform IWT denoising on the noise-containing part to maximally retain the signal components. The block diagram of the proposed algorithm is shown in Figure 1.

First, the source signal $s(n)$ interferes with noise in the transmission process, and the receiver receives the mixed signal $x(n)$. The received signal $x(n)$ is decomposed using ICEEMDAN to obtain the first-round decomposition components denoted by $h_{1}^{(i)}(n)$. For the first dividing point 


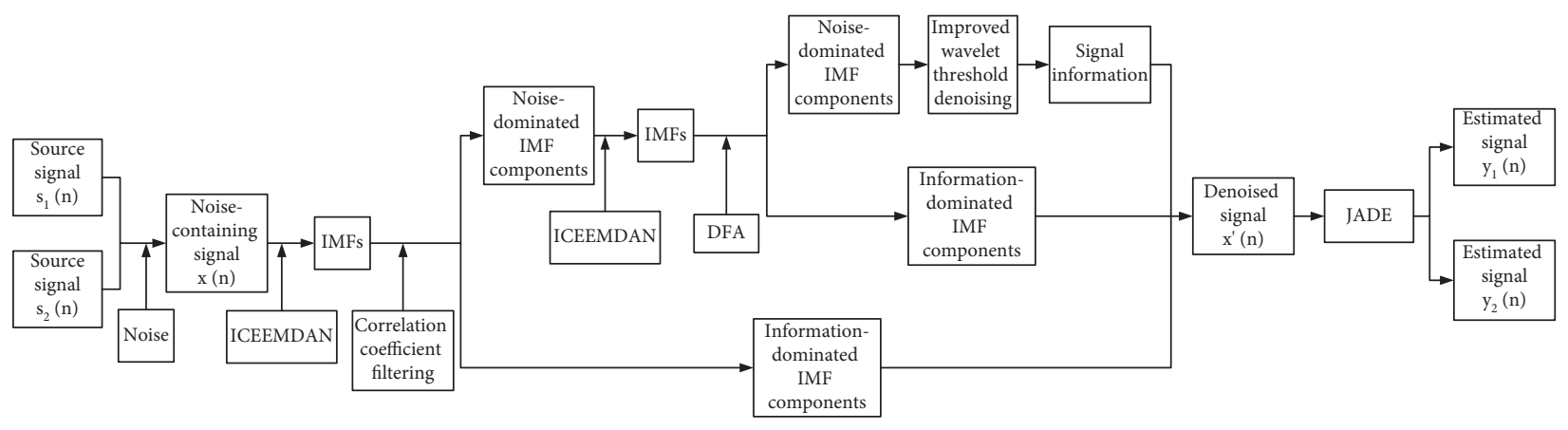

FIgURE 1: Block diagram of the algorithm proposed in this study.

calibration, the IMF components decomposed for the first time are preliminarily screened by using the principle of correlation coefficient [36].

The estimated value $\widetilde{x}_{m}(n)$ of the signal can be defined as

$$
\tilde{x}_{m}(n)=x(n)-\sum_{i=1}^{m} h_{1}^{(i)}(n) .
$$

Correlation coefficients are calculated as

$$
\operatorname{COR}(m)=\frac{\sum_{j=1}^{N} x(j) \tilde{x}_{m}(j)}{\sqrt{\sum_{j=1}^{N} x^{2}(j)} \sqrt{\sum_{j=1}^{N} \tilde{x}_{m}^{2}(j)}},
$$

where $N$ is the signal length, and $M$ is the value at which the correlation coefficient begins falls below a certain constant $C$, i.e., a threshold dividing the noise-dominated IMF components and the information-dominated IMF components generated in the first round of decomposition. Typically, $C \in[0.75,0.85]$. In this study, $C$ is set to 0.80 . Now,

$$
m=\arg \operatorname{last}_{1 \leq m \leq M}\{\operatorname{COR}(m)>0.8\}+1,
$$

where $M$ is the number of IMF components generated in the first decomposition round. Taking the $m^{\text {th }}$ IMF component as the dividing point, the previous IMF components are the noise-dominated IMF components, and the rest are the information-dominated IMF components. In the first decomposition, most of the signal information has been filtered out.

Then, the second-round ICEEMDAN decomposition is performed on the noise-dominated IMF components to obtain the IMF components $h_{2}^{(i)}(n)$ of the second-round decomposition. Because most of the signal information has been filtered out in the first decomposition, the second dividing point calibration needs to be more accurate. The DFA algorithm can better solve the problem of calibration ambiguity. Therefore, we choose to use the DFA algorithm to calibrate the dividing point of the second decomposition, and the scaling function $\alpha_{2}^{(i)}$ is obtained based on the Hurst index of each IMF component. The dividing point $k$ separating the noise-dominated IMF components from the information-dominated ones generated in the second-round decomposition is obtained. Then, IWT denoising is performed on the noise-dominated IMF components of the second-round decomposition to obtain the signal information part $u^{(i)}(n)$.

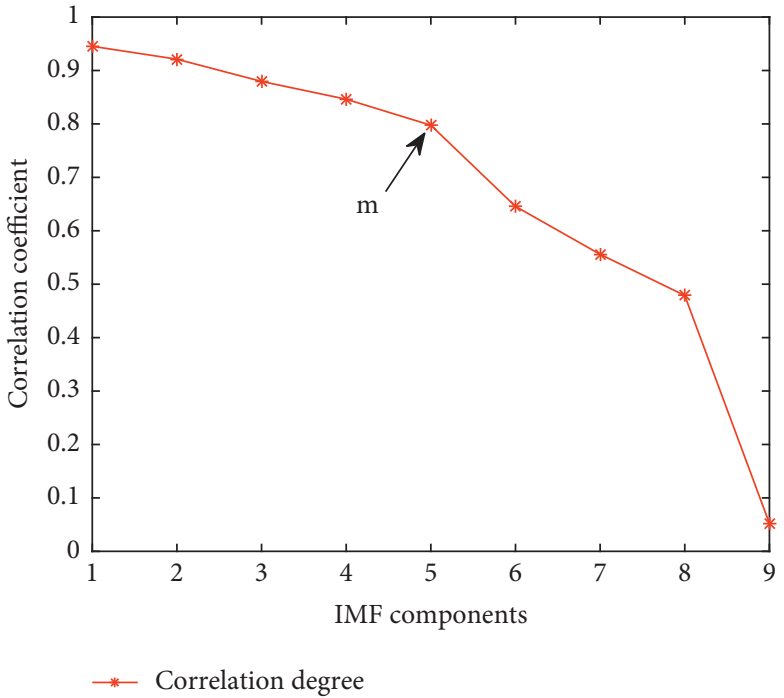

FIGURE 2: Correlation coefficient values of the IMF components after the signal undergoes ICEEMDAN processing when SNR is $5 \mathrm{~dB}$.

Finally, signal reconstruction is performed using the information-dominated IMF components obtained in the first and second decomposition rounds, as well as the signal information resulting from denoising the noise-dominated IMF components in the second-round decomposition.

$$
x^{\prime}(n)=\sum_{i=m+1}^{M-1} h_{1}^{(i)}(n)+r_{1}(n)+\sum_{i=k+1}^{K-1} h_{2}^{(i)}(n)+r_{2}(n)+\sum_{i=1}^{k} u^{(i)}(n)
$$

where $K$ is the number of IMF components generated in the second-round decomposition, and $r_{1}(n)$ and $r_{2}(n)$ are the residuals generated in the first and second decomposition rounds, respectively. The reconstructed signal undergoes a BSS separation process to obtain the estimated values $y(n)$ of the source signals. In this work, JADE is used as the BSS algorithm.

\section{Performance Analysis}

We use the "heavy sine" test signal with a length of 1,024 in MATLAB as the source signal $s_{1}(t)$. Set $s_{2}(t)=\sin (\pi / 32 t)+\sin (\pi / 25 t)+\sin (\pi / 18 t)$. The mixing matrix is $A=\left[\begin{array}{cc}\cos (\pi / 4) & -\sqrt{10} \sin (\pi / 4) \\ \sin (\pi / 4) & \sqrt{10} \cos (\pi / 4)\end{array}\right]$. An additive 

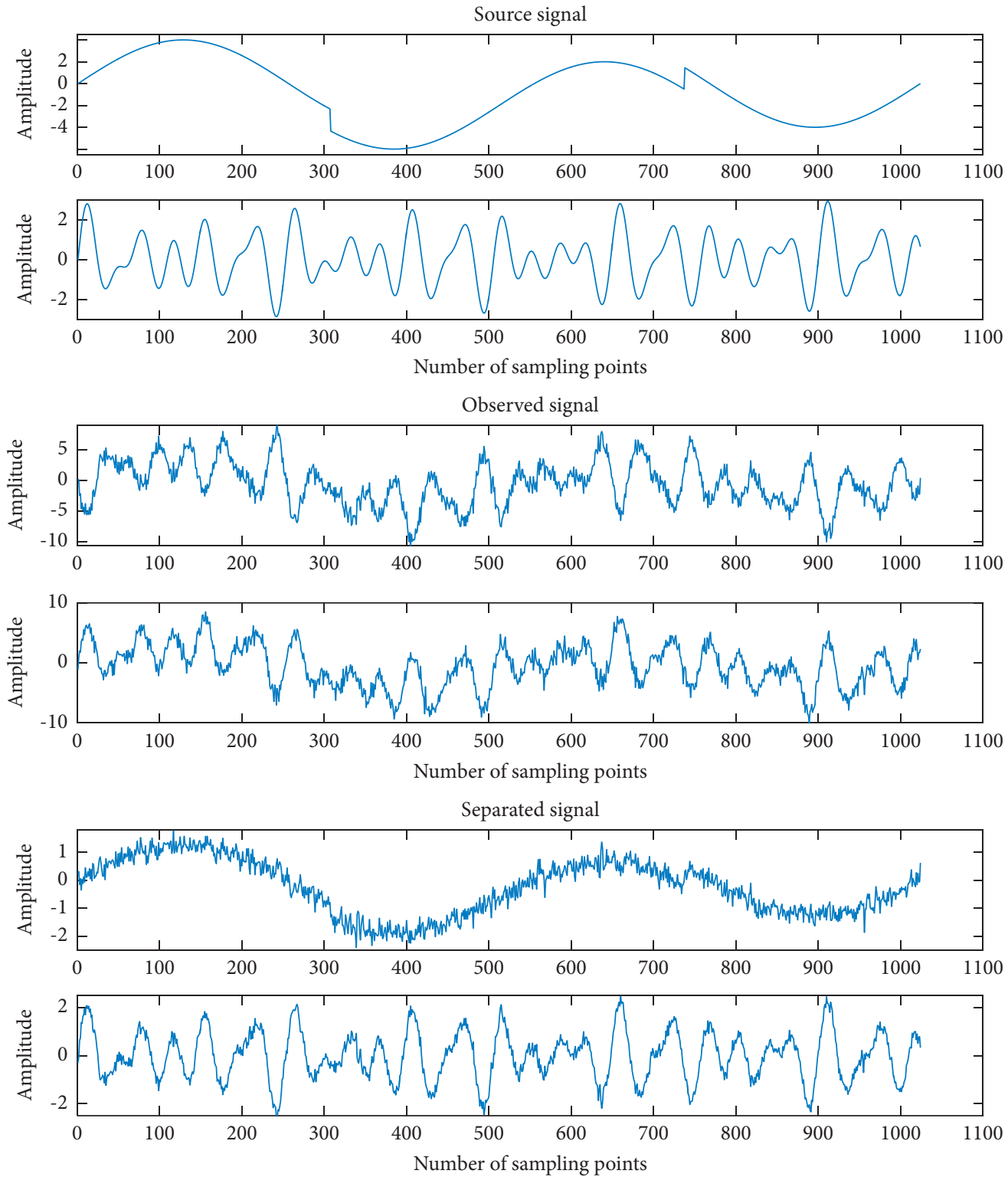

FIGURE 3: Source signal, observed signal, and separated signal when SNR is $1 \mathrm{~dB}$.

Gaussian white noise is added to the mixed signal, and SNR ranges between 0 and $15 \mathrm{~dB}$.

During the first-round decomposition, the generated IMF components are screened based on the correlation coefficient to obtain the point $m$ dividing noise-dominated from information-dominated IMF components. The correlation coefficient value diagram is shown in Figure 2. It can be seen from the figure that when SNR equals $5 \mathrm{~dB}, m$ equals five (i.e., 1-5 IMF components are noise-dominated, whereas 6-9 IMF components are information-dominated).

The noise-dominated IMF components are useless in the traditional EMD denoising algorithms. However, implementing the hierarchical decomposition and denoising strategy allows them to be decomposed once again to extract signal information. First, the Hurst index of each IMF component generated in the second-round decomposition is calculated using the DFA algorithm, and the IMF component dividing point is obtained. Then, the noise-dominated IMF components are denoised using the IWT denoising algorithm to obtain useful signal information. Next, signal reconstruction is performed following equation (21) to obtain the reconstructed signal. Finally, JADE is used to decompose the reconstructed signal, obtaining the source signal. Figure 3 shows the source signal, the observed signal, and the separated signal yielded by the proposed algorithm when SNR equals $1 \mathrm{~dB}$.

In this study, the performance index (PI) [37] is used as a metric to evaluate the separation effect. PI is defined as 


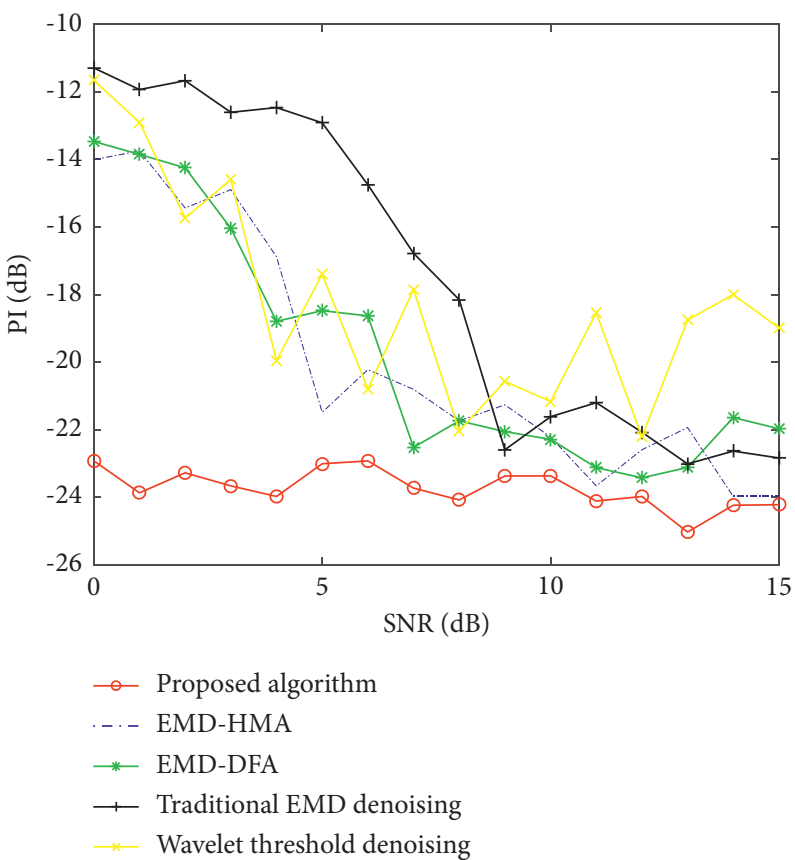

FIGURE 4: Comparison of the signal separation's PI values of the proposed algorithm and the considered algorithms.

$$
P I(C)=\frac{1}{2(N-1)}\left[\sum_{i=1}^{N}\left(\sum_{j=1}^{N} \frac{\left|c_{i j}\right|^{2}}{\max _{k}\left|c_{i k}\right|^{2}}-1\right)+\sum_{j=1}^{N}\left(\sum_{i=1}^{N} \frac{\left|c_{i j}\right|^{2}}{\max _{k}\left|c_{k j}\right|^{2}}-1\right)\right],
$$

where $N=2, C$ is the system matrix, and $c_{i j}$ is the $j^{\text {th }}$ element in the $i^{\text {th }}$ row of C. As defined, the PI value cannot be less than 0 . When $C$ is a generalized permutation matrix, PI reaches its minimum value (i.e., zero). The closer the PI is to 0 , the better the separation performance of the algorithm and the higher the signal separation accuracy.

This study compares the proposed algorithm with EMD-HMA [24], EMD-DFA [23], wavelet threshold denoising[38], and traditional EMD denoising method [39] (Figure 4).

As shown in Figure 4, the proposed algorithm achieves a better separation effect than other considered algorithms, exhibiting a salient advantage under the condition of low SNR. When SNR is $1 \mathrm{~dB}$, the PI value of the proposed algorithm is $-23.87 \mathrm{~dB}$, which amounts to an improvement of $10.12 \mathrm{~dB}$ compared with the EMD-HMA's PI $(-13.75 \mathrm{~dB})$. The difference in the traditional EMD-based algorithms' performance stems from a signal information loss due to discarding the noise-dominated IMF components, resulting in incomplete signal recovery. To mitigate the limitations of EMD-DFA and traditional EMD denoising methods, the EMD-HMA algorithm proposed in [24] adopts a hierarchical decomposition strategy. However, this method discards the noise-dominated IMF components generated in the second-round decomposition, thus leading to incomplete signal recovery. Furthermore, the wavelet threshold denoising method has poor adaptability due to the intrinsic limitations of the wavelet basis. Based on an analysis of the deficiencies of the existing algorithms, this work adopts a hierarchical decomposition strategy for the proposed algorithm. With this arrangement, the noise-dominated IMF components generated in the second-round decomposition are processed using the IWT denoising method, maximally retaining the signal information and endowing the algorithm with a degree of adaptability.

\section{Conclusion}

The BSS algorithm separates the source signals from a mix of signals. However, noise seriously affects signal separation. Given this problem, this work carried out an in-depth study on the problem of BSS under a noisy condition and proposed a method that mitigates the shortcomings of the existing algorithms. The new BSS method features hierarchical decomposition and denoising strategy based on ICEEMDAN and IWT and can retain the signal information to the maximum extent, improving the separation effect. The simulation analysis results indicate that the proposed algorithm is superior to the existing algorithms. When SNR is $1 \mathrm{~dB}$, the proposed algorithm achieves a signal separation effect of at least $10.12 \mathrm{~dB}$ higher than the existing algorithms, significantly improving the signal separation effect under low SNR conditions.

\section{Data Availability}

No data were used to support this study. 


\section{Conflicts of Interest}

The authors declare that they have no conflicts of interest.

\section{Acknowledgments}

This work was supported by the National Natural Science Foundation of China (62001516).

\section{References}

[1] P. Comon, "Independent component analysis, a new concept?" Signal Processing, vol. 36, no. 3, pp. 287-314, 1994.

[2] G. R. Naik and W. Wang, Blind Source Separation: Advances in Theory, Algorithms and Applications," Ser. Signals and Communication Technology, Springer, Berlin, Germany, 2014.

[3] B. Du, S. Wang, C. Xu, N. Wang, L. Zhang, and D. Tao, "Multi-task learning for blind source separation," IEEE Transactions on Image Processing, vol. 27, no. 9, pp. 42194231, 2018.

[4] L. Di Persia, M. Yanagida, H. L. Rufiner, and D. Milone, "Objective quality evaluation in blind source separation for speech recognition in A real room," Signal Processing, vol. 87, no. 8, pp. 1951-1965, 2007.

[5] N. Hassan and D. A. Ramli, "A comparative study of blind source separation for bioacoustics sounds based on FastICA, PCA and NMF," Procedia Computer Science, vol. 126, pp. 363-372, 2018.

[6] H. Wu, D. Li, M. Lu, and Y. Zeng, "fMRI activations via lowcomplexity second-order inverse-sparse-transform blind separation," Digital Signal Processing, vol. 117, Article ID 103137, 2021.

[7] B. Ghosh, M. Motagh, M. H. Haghighi, M. S. Vassileva, T. R. Walter, and S. Maghsudi, "Automatic detection of volcanic unrest using blind source separation with a minimum spanning tree based stability analysis," Ieee Journal of Selected Topics in Applied Earth Observations and Remote Sensing, vol. 14, pp. 7771-7787, 2021.

[8] R. R. Vázquez, H. Velez-Perez, R. Ranta, V. Louis, D. Maquin, and L. Maillard, "Blind source separation, wavelet denoising and discriminant analysis for EEG artefacts and noise cancelling," Biomedical Signal Processing and Control, vol. 7, no. 4, pp. 389-400, 2012.

[9] Z. H. Zhang, F. Min, G. S. Chen, S. Shen, Z. Wen, and X. Zhou, "Tri-partition state alphabet-based sequential pattern for multivariate time series," Cognitive Computation, pp. 1-19, 2021.

[10] X. Ran, X. Zhou, M. Lei, W. Tepsan, and W. Deng, “A novel K-means clustering algorithm with a noise algorithm for capturing urban hotspots," Applied Sciences, vol. 11, no. 23, Article ID 11202, 2021.

[11] H. Cui, Y. Guan, H. Chen, and W. Deng, "A novel advancing signal processing method based on coupled multi-stable stochastic resonance for fault detection," Applied Sciences, vol. 11, no. 12, p. 5385, 2021.

[12] W. Deng, X. Zhang, Y. Zhou et al., "An enhanced fast nondominated solution sorting genetic algorithm for multi-objective problems," Information Sciences, vol. 585, pp. 441-453, 2022.

[13] E. Q. Wu, M. C. Zhou, D. Hu et al., "Self-paced dynamic infinite mixture model for fatigue evaluation of pilots' brains," IEEE Transactions on Cybernetics, 2020.

[14] P. Lei, M. Chen, and J. Wang, "Speech enhancement for invehicle voice control systems using wavelet analysis and blind source separation," IET Intelligent Transport Systems, vol. 13, no. 4, pp. 693-702, 2019.

[15] K. Wang, X. Chen, L. Wu, X. Zhang, X. Chen, and Z. J. Wang, "High-density surface EMG denoising using independent vector analysis," IEEE Transactions on Neural Systems and Rehabilitation Engineering, vol. 28, no. 6, pp. 1271-1281, 2020.

[16] H. Snoussi and J. Idier, "Bayesian blind separation of generalized hyperbolic processes in noisy and underdeterminate mixtures," IEEE Transactions on Signal Processing, vol. 54, no. 9, pp. 3257-3269, 2006.

[17] L. Hongyan and R. Guanglong, "Blind separation of noisy mixed speech signals based independent component analysis," in Proceedings of the 2010 1st International Conference on Pervasive Computing, Signal Processing and Applications, pp. 586-589, Harbin, China, September 2010.

[18] F. Gu, H. Zhang, W. Wang, and S. Wang, "An expectationmaximization algorithm for blind separation of noisy mixtures using Gaussian mixture model," Circuits, Systems, and Signal Processing, vol. 36, no. 7, pp. 2697-2726, 2017.

[19] A. Weiss and A. Yeredor, "A maximum likelihood-based minimum mean square error separation and estimation of stationary Gaussian sources from noisy mixtures," IEEE Transactions on Signal Processing, vol. 67, no. 19, pp. 5032$5045,2019$.

[20] N. E. Huang, Z. Shen, S. R. Long et al., “The empirical mode decomposition and the Hilbert spectrum for nonlinear and non-stationary time series analysis," Proceedings of the Royal Society of London. Series A: Mathematical, Physical and Engineering Sciences, vol. 454, no. 1971, pp. 903-995, 1998.

[21] T. George and T. D. Xenos, "Signal de-noising using empirical mode decomposition and higher order statistics," International Journal of Signal Processing, Image Processing and Pattern Recognition, vol. 4, no. 2, 2011.

[22] Y. Kopsinis and S. McLaughlin, "Development of EMD-based denoising methods inspired by wavelet thresholding," IEEE Transactions on Signal Processing, vol. 57, no. 4, pp. 13511362, April 2009.

[23] X. Y. Qian, G. F. Gu, and W. X. Zhou, "Modified detrended fluctuation analysis based on empirical mode decomposition for the characterization of anti-persistent processes," Physica A: Statistical Mechanics and Its Applications, vol. 390, no. 2324, pp. 4388-4395, 2011.

[24] Y. Zhou, B. W.-K. Ling, X. Mo, Y. Guo, and Z. Tian, "Empirical mode decomposition-based hierarchical multiresolution analysis for suppressing noise," IEEE Transactions on Instrumentation and Measurement, vol. 69, no. 4, pp. 1833-1845, 2020.

[25] Z. Wu and N. E. Huang, "Ensemble empirical mode decomposition: a noise-assisted data analysis method," Advances in Adaptive Data Analysis, vol. 01, no. 01, pp. 1-41, 2009.

[26] J. R. Yeh, J. S. Shieh, and N. E. Huang, "Complementary ensemble empirical mode decomposition: a novel noise enhanced data analysis method," Advances in Adaptive Data Analysis, vol. 2, no. 2, pp. 135-156, 2010.

[27] M. E. Torres, M. A. Colominas, G. Schlotthauer, and P. Flandrin, "A complete ensemble empirical mode decomposition with adaptive noise," in Proceedings of the 2011 IEEE International Conference on Acoustics, Speech and Signal Processing (ICASSP), pp. 4144-4147, Prague, Czech Republic, May 2011.

[28] Y. Li, L. Wang, X. Li, and X. Yang, “A novel linear spectrum frequency feature extraction technique for warship radio noise based on complete ensemble empirical mode 
decomposition with adaptive noise, duffing chaotic oscillator, and weighted-permutation entropy," Entropy, vol. 21, no. 5, p. 507, 2019.

[29] Y.-x. Li and L. Wang, "A novel noise reduction technique for underwater acoustic signals based on complete ensemble empirical mode decomposition with adaptive noise, minimum mean square variance criterion and least mean square adaptive filter," Defence Technology, vol. 16, no. 3, pp. 543-554, 2020.

[30] M. A. Colominas, G. Schlotthauer, and M. E. Torres, "Improved complete ensemble EMD: a suitable tool for biomedical signal processing," Biomedical Signal Processing and Control, vol. 14, pp. 19-29, 2014.

[31] C.-K. Peng, S. V. Buldyrev, S. Havlin, M. Simons, H. E. Stanley, and A. L. Goldberger, "Mosaic organization of DNA nucleotides," Physical Review A, vol. 49, no. 2, pp. 1685-1689, 1994.

[32] Q. Song, S. Zhao, and M. Wang, "On the accuracy of fault diagnosis for rolling element bearings using improved DFA and multi-sensor data fusion method," Sensors, vol. 20, no. 22, 2020.

[33] Y. Wang, C. Xu, Y. Wang, and X. Cheng, "A comprehensive diagnosis method of rolling bearing fault based on CEEMDAN-DFA-improved wavelet threshold function and QPSOMPE-SVM," Entropy, vol. 23, no. 9, 2021.

[34] A.-O. Boudraa and J.-C. Cexus, "EMD-based signal filtering," IEEE Transactions on Instrumentation and Measurement, vol. 56, no. 6, pp. 2196-2202, 2007.

[35] S. G. Mallat, "A theory for multiresolution signal decomposition: the wavelet representation," Fundamental Papers in Wavelet Theory, Princeton University Press, Princeton, NJ, USA, pp. 494-513, 2009.

[36] Y. Duan and C. Song, "Relevant modes selection method based on Spearman correlation coefficient for laser signal denoising using empirical mode decomposition," Optical Review, vol. 23, no. 6, pp. 936-949, 2016.

[37] S. Amiri, A. Cichocki, and H. H. Yang, "A new learning algorithm for blind source separation," Advances in Neural Information Processing Systems, vol. 8, pp. 757-763, 1996.

[38] I. M. Johnstone and B. W. Silverman, "Wavelet threshold estimators for data with correlated noise," Journal of the Royal Statistical Society, vol. 59, 1997.

[39] A. O. Boudraa, J. C. Cexus, and Z. Saidi, "EMD-based signal noise reduction," International Journal of Signal Processing, vol. 1, no. 1, pp. 33-37, 2004. 